\title{
Simple low-frequency beam pickup
}

\author{
A. Novokhatski* and S. Heifets \\ Stanford Linear Accelerator Center, Stanford University, Stanford, California 94309, USA
}

\section{A. Aleksandrov}

Oak Ridge National Laboratory, Spallation Neutron Source, Oak Ridge, Tennessee 37830, USA

(Received 30 April 2008; published 12 November 2008)

\begin{abstract}
Detection of the field induced by a beam outside of the beam pipe can be used as a beam diagnostic. Wires placed in longitudinal slots in the outside wall of the beam pipe can be used as a beam pickup. This has a very small beam-coupling impedance and avoids complications of having a feedthrough. The signal can be reasonably high at low frequencies. We present a field waveform at the outer side of a beam pipe, obtained as a result of calculations and measurements. We calculate the beam-coupling impedance due to a long longitudinal slot in the resistive wall and the signal induced in a wire placed in such a slot and shielded by a thin screen from the beam. These results should be relevant for impedance calculations of the slot in an antechamber and for slots in the PEP-II distributed ion pump screens. The design of the lowfrequency beam position monitor is very simple. It can be used in storage rings, synchrotron light sources, and free electron lasers, like LINAC coherent light source.
\end{abstract}

DOI: 10.1103/PhysRevSTAB.11.114401

PACS numbers: 29.27.Bd, 29.20.db, 41.60.- m, 52.59.-f

\section{INTRODUCTION}

The electromagnetic (EM) field induced by a beam outside of a thin beam pipe may be quite noticeable. The analytical solution for electromagnetic fields in a round beam pipe in the frequency domain can be found elsewhere [1]. The field waveform can be determined by numerically solving the wave equations in the time domain [2]. As a numerical example, Fig. 1 shows the time profile of the field induced by a bunch on the inner side (pancake thin red line) and on the outer side (blue line) of a stainless-steel tube. The bunch length is $10 \mathrm{~mm}$, the tube radius is $5 \mathrm{~mm}$, and the wall thickness is $0.1 \mathrm{~mm}$. One can see that the field amplitude outside of the pipe decreases by only a factor of 100.

Another example is given in Fig. 2 for an aluminum chamber with a radius of $2.5 \mathrm{~mm}$ and with a tube thickness of $0.5 \mathrm{~mm}$ (parameters of the LINAC coherent light source [3], round chamber). The signal outside of the pipe in this case may reach an amplitude of $35 \mathrm{~V} / \mathrm{m}$ for a $1 \mathrm{nC}$ bunch.

In both examples, the main contribution to the signal is given by the low-frequency modes which can penetrate through the wall. Such frequencies for short bunches are much lower than the width of the bunch spectrum. Therefore, the signal is practically independent on the bunch length which simplifies any design of the beam position monitor (BPM) electronics. Another common feature of both results is the time delay between the signals on the inner and outer sides defined by the diffusion time of

*novo@slac.stanford.edu the magnetic field through the wall (about $3 \mathrm{~ns}$ in Fig. 1 and 200 ns in Fig. 2).

The field outside of the beam pipe can be detected and used to build a BPM without any feedthrough thereby preserving the smooth beam pipe wall seen by the beam. An idea of a BPM based on the detection of the EM field behind a thin foil was suggested long ago [4]. Based on this approach, a low impedance BPM was proposed and tested by one of the authors (A. A.) for the VEPP-5 collider, a Bfactory project planned to be built in Novosibirsk [5]. To prove the feasibility of the approach an experimental model was built. The experimental signal measured the outside of the beam pipe with a $15 \mathrm{~mm}$ inner radius [5] is

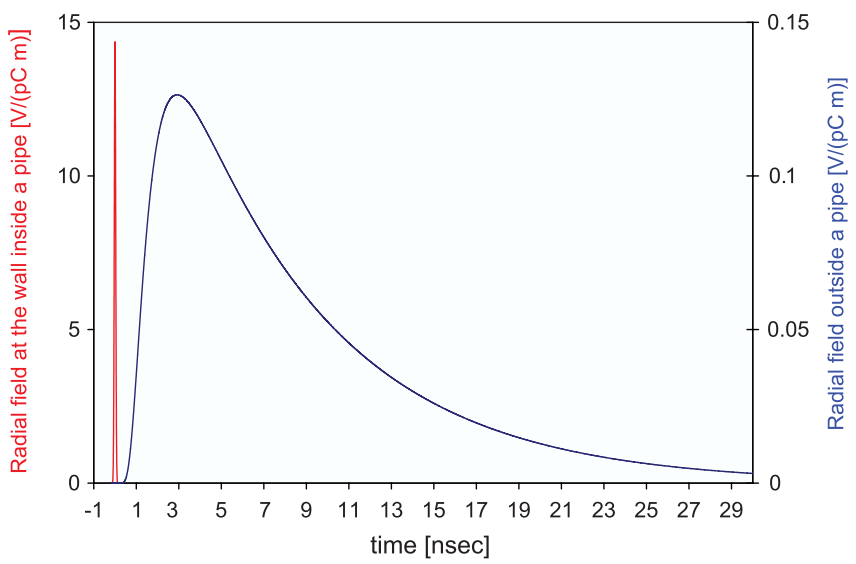

FIG. 1. (Color) The radial component of the $E$ field on the inner (red line) and outer (blue line) sides of a beam pipe. Scales for the fields are on the left and right plot side. 


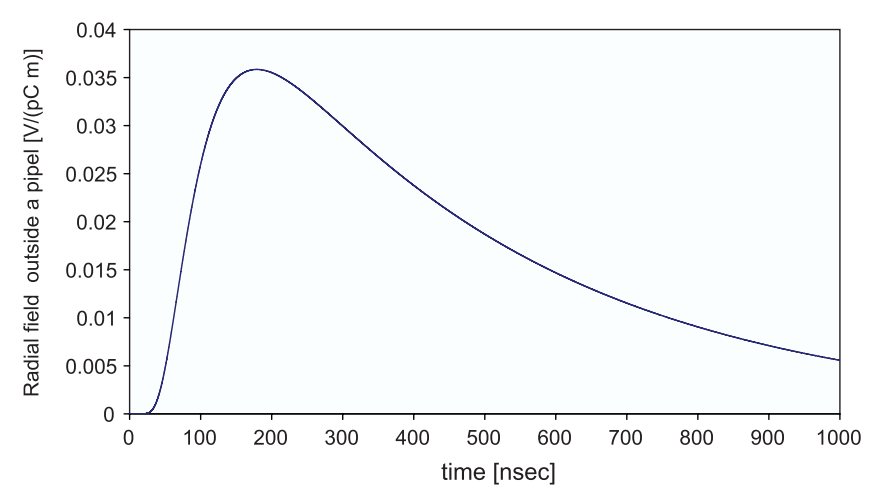

FIG. 2. Field outside of the Al $0.5 \mathrm{~mm}$ round beam pipe.

shown in Fig. 3. At that time, the full solution for EM fields was not obtained but simple estimates were used to derive the signal amplitude and duration. The dipole mode of the beam EM field was simulated by a short pulse propagating in a two wire transmission line. The transmission line was inserted into an aluminum pipe with the central part of the pipe replaced with a $50 \mu \mathrm{m}$ thick stainless-steel foil. The magnetic field penetrating through the foil was measured using a 12 turn coil with a $2 \times 2 \mathrm{~cm}$ cross section. An oscilloscope snapshot of the current pulse in the transmission line and the signal measured by the coil are shown in Figs. 3(a) and 3(b), respectively [5]. The measured signal amplitude and duration were in good agreement with expected values.

This kind of a BPM could be used in a free-electron lasers like LCLS where the wall thickness can be as small as $0.5 \mathrm{~mm}$ [3]. In general, a BPM can be made from a loop of wire set into a thin longitudinal groove (or several grooves) in the outer side of the beam pipe wall, see Fig. 4. For simplicity, we consider a round beam pipe denoting the inner radius $a$, the thickness of a screen $\Delta$, and the wall conductivity $\sigma_{w}$. In the first approximation, the effects of several wires are additive because the pipe walls provide natural screening reducing the cross talk between wires. Consequently, it suffices to consider the effect of a single wire.

\section{EM FIELDS IN A BEAM PIPE WITH A SLOT}

Let us begin with calculations of the EM fields in a pipe with a slot using Maxwell's equations for a particle moving in a round beam pipe along the $z$ axes with the offset $r_{0}$ and velocity $v$. Assuming a time dependence of the form $e^{-i \omega t}$, equations for the $\omega$-frequency components of EM fields generated by the particle are

$$
\nabla \times E=\frac{i \omega}{c} B, \quad \nabla \times B=\frac{4 \pi}{c}\left(j^{b}+\sigma E\right)-\frac{i \omega}{c} E,
$$

where $\sigma$ is the wall conductivity considered a constant over $\omega, \rho_{b}$ is the particle density, and $j^{b}$ is the current. The second equation can be rewritten introducing $D=\epsilon E$ to be

$$
\nabla \times B=\frac{4 \pi}{c} j^{b}-\frac{i \omega}{c} D, \quad \epsilon=1+i \frac{4 \pi \sigma}{\omega} .
$$

The first of the two equations in (1) gives $\operatorname{div} B=0$, and from Eq. (2) and the continuity equation

$$
-i \omega \rho^{b}+\operatorname{div} j^{b}=0
$$

it follows that $\operatorname{div} D=4 \pi \rho^{b}$.

In cylindrical coordinates with the polar axis along the beam pipe axis,

$$
\rho^{b}=\frac{e}{v r} \delta\left(r-r_{0}\right) \delta(\phi) e^{i \omega z / v}
$$

The wave equation follows from $\nabla \times \nabla \times B=$ $\nabla(\nabla B)-\triangle B$ and Maxwell's equations. In the regions of constant $\epsilon$,

$$
\triangle B+\left(\frac{\omega}{c}\right)^{2} \epsilon B=-\frac{4 \pi}{c} \nabla \times j^{b},
$$

where the current $j^{b}=\hat{z} v \rho^{b}$ has only a $z$ component along the beam pipe, and
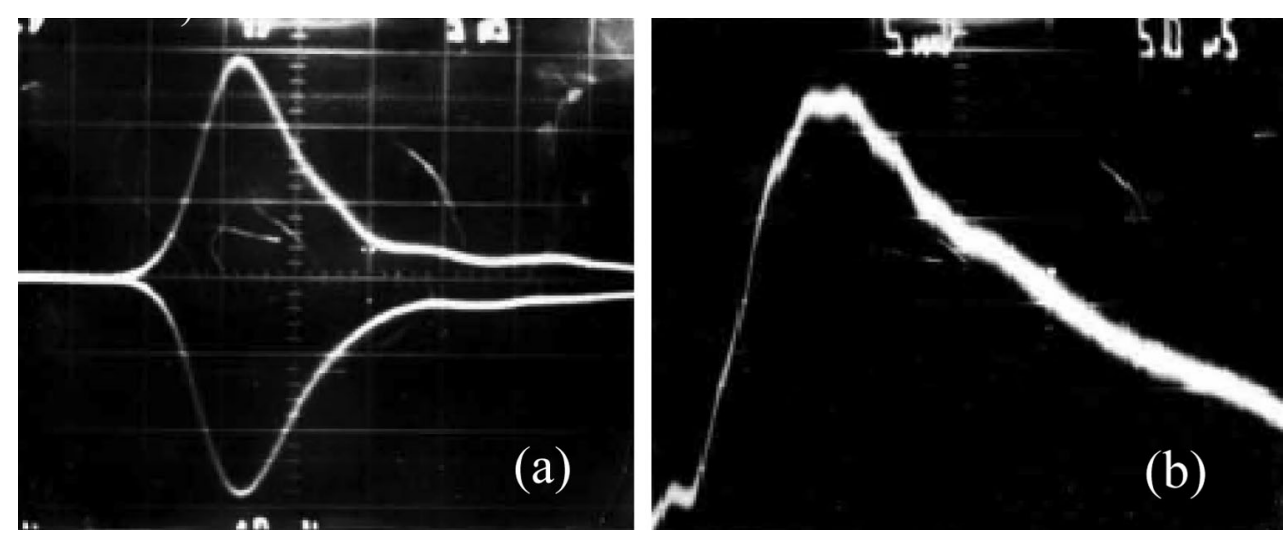

FIG. 3. (a) The signal from the transmission line terminating resistors. The horizontal scale is $5 \mathrm{~ns} / \mathrm{div}$ and the vertical scale is $20 \mathrm{~V} /$ div. (b) The signal from the measuring coil. The horizontal scale is $50 \mathrm{~ns} /$ div and the vertical scale is $20 \mathrm{~V} /$ div. 


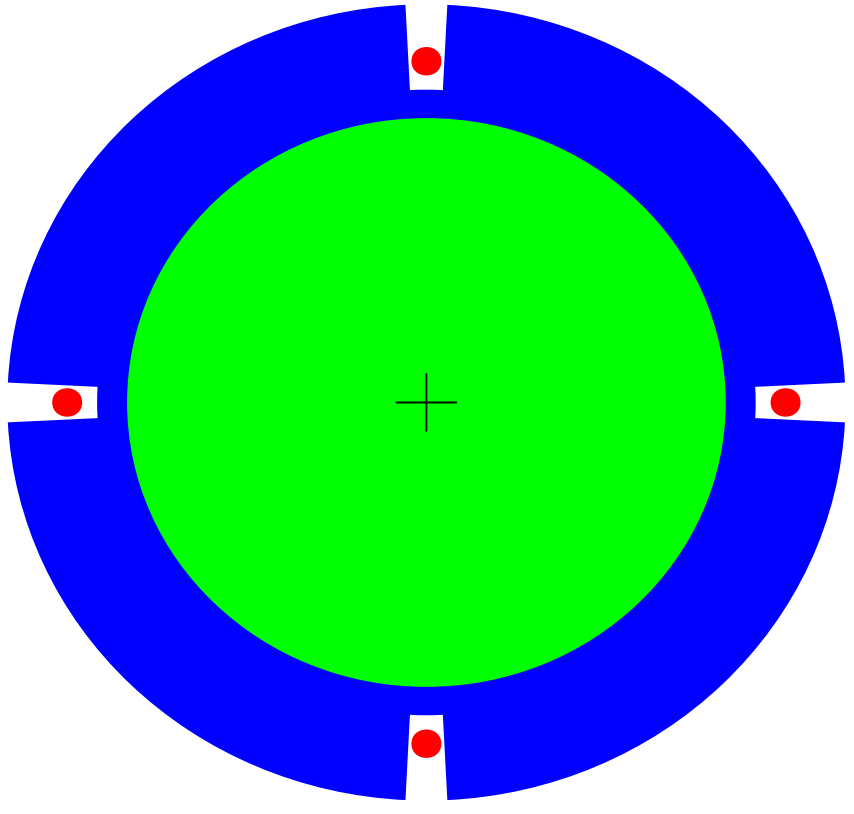

FIG. 4. (Color) Sketch of the BPM design.

$$
\left(\nabla \times j^{b}\right)_{r}=\frac{v}{r}\left(\frac{\partial \rho}{\partial \phi}\right), \quad\left(\nabla \times j^{b}\right)_{\phi}=-v\left(\frac{\partial \rho}{\partial r}\right),
$$$$
\left(\nabla \times j^{b}\right)_{z}=0 .
$$

At the boundaries, where $\epsilon$ changes its value, tangential components of $E$ and $B$ have to be continuous. Then the normal components of $D$ and $B$ are continuous automatically.

Let us expand $B(r, \phi, z)$ over the azimuthal harmonics and assume a dependence on $z$ of the form $e^{i \omega z / v}$,

$$
\begin{aligned}
B(r, \phi, z)= & e^{i \omega z / v} \sum_{m=-\infty}^{\infty}\left[\hat{r} B_{m}^{r}(r)+\hat{\phi} B_{m}^{\phi}(r)\right. \\
& \left.+\hat{z} B_{m}^{z}(r)\right] e^{i m \phi},
\end{aligned}
$$

where $\hat{r}(\phi), \hat{\phi}(\phi)$, and $\hat{z}$ are unit vectors. For a round beam pipe the only direction breaking azimuthal symmetry is the direction to the slot. We assume below that $\phi=0$ corresponds to this direction and refer to the plane of a slot as the horizontal plane.

Equation (5), rewritten for the components $B_{m}^{ \pm}(r)=$ $B_{m}^{r} \pm i B_{m}^{\phi}$ and $B_{m}^{z}$ now takes the form

$$
\begin{aligned}
& {\left[\frac{1}{r} \frac{\partial}{\partial r} r\left(\frac{\partial}{\partial r}\right)-\left(\frac{m \pm 1}{r}\right)^{2}-\left(\frac{\omega}{v}\right)^{2}\left(1-\frac{v^{2}}{c^{2}} \epsilon\right)\right] B_{m}^{ \pm}(r)} \\
& \quad=-\frac{4 \pi i v}{c}\left(\frac{m \rho_{m}(r)}{r} \mp \frac{\partial \rho_{m}(r)}{\partial r}\right), \\
& {\left[\frac{1}{r} \frac{\partial}{\partial r} r\left(\frac{\partial}{\partial r}\right)-\left(\frac{m}{r}\right)^{2}-\left(\frac{\omega}{v}\right)^{2}\left(1-\frac{v^{2}}{c^{2}} \epsilon\right)\right] B_{m}^{z}(r)=0,}
\end{aligned}
$$

where

$$
\rho_{m}(r)=\frac{e}{2 \pi v r} \delta\left(r-r_{0}\right)
$$

Inside the beam pipe, $\epsilon=1$. In the ultrarelativistic case, the equations in (8) simplify to

$$
\begin{gathered}
{\left[\frac{1}{r} \frac{\partial}{\partial r} r\left(\frac{\partial}{\partial r}\right)-\left(\frac{m \pm 1}{r}\right)^{2}\right] B_{m}^{ \pm}(r)} \\
=-4 \pi i\left(\frac{m \rho_{m}(r)}{r} \mp \frac{\partial \rho_{m}(r)}{\partial r}\right) \\
{\left[\frac{1}{r} \frac{\partial}{\partial r} r\left(\frac{\partial}{\partial r}\right)-\left(\frac{m}{r}\right)^{2}\right] B_{m}^{z}(r)=0 .}
\end{gathered}
$$

In the region of $r<r_{0}$ the solution does not contain a singularity at $r \rightarrow 0$ and has to be matched with the solution in the region $r_{0}<r<a$. The conditions for matching at $r=r_{0}$ are defined by the right-hand side (RHS) of Eq. (10):

$$
\begin{aligned}
B_{m}^{ \pm,>}\left(r_{0}\right)-B_{m}^{ \pm,}<\left(r_{0}\right) & = \pm \frac{i e Z_{0}}{2 \pi r_{0}} \\
{\left[\frac{\partial B_{m}^{ \pm,>}(r)}{\partial r}-\frac{\partial B_{m}^{ \pm,<}(r)}{\partial r}\right]_{r=r_{0}} } & =-\frac{i e Z_{0}(m \pm 1)}{2 \pi r_{0}^{2}} .
\end{aligned}
$$

Here $Z_{0}=4 \pi / c=120 \pi \Omega$.

The fields at $r<r_{0}$ are

$$
\begin{aligned}
& B_{m}^{+,<}(r)=c_{m}^{+}\left(\frac{r}{r_{0}}\right)^{m+1}, \\
& B_{m}^{-,<}(r)=\left(\frac{i e Z_{0}}{2 \pi r_{0}}+c_{m}^{-}\right)\left(\frac{r}{r_{0}}\right)^{m-1}, \\
& B_{m}^{z,<}(r)=i \frac{m+1}{k r_{0}} c_{m}^{+}\left(\frac{r}{r_{0}}\right)^{m} \text {. } \\
& E_{m}^{r,<}(r)=-\frac{i}{2 k^{2} r_{0}^{2}}\left(\frac{r}{r_{0}}\right)^{m-1}\left\{c_{m}^{+}\left(2 m+2 m^{2}+k^{2} r^{2}\right)\right. \\
& \left.-c_{m}^{-} k^{2} r_{0}^{2}-i e Z_{0} k^{2} r_{0}\right\} \text {, } \\
& E_{m}^{\phi,<}(r)=\frac{1}{2 k^{2} r_{0}^{2}}\left(\frac{r}{r_{0}}\right)^{m-1}\left\{c_{m}^{+}\left(2 m+2 m^{2}-k^{2} r^{2}\right)\right. \\
& \left.-c_{m}^{-} k^{2} r_{0}^{2}-i e Z_{0} k^{2} r_{0}\right\} \text {, } \\
& E_{m}^{z,}<(r)=\frac{m+1}{k r_{0}}\left(\frac{r}{r_{0}}\right)^{m} c_{m}^{+} \text {. }
\end{aligned}
$$

The fields at $r_{0}<r<a$ are

$$
\begin{gathered}
B_{m}^{+,>}(r)=c_{m}^{+}\left(\frac{r}{r_{0}}\right)^{m+1}+\frac{i Z_{0}}{2 \pi r_{0}}\left(\frac{r_{0}}{r}\right)^{m+1}, \\
B_{m}^{-,>}(r)=c_{m}^{-}\left(\frac{r}{r_{0}}\right)^{m-1}, \quad B_{m}^{z,>}(r)=i \frac{m+1}{k r_{0}} c_{m}^{+}\left(\frac{r}{r_{0}}\right)^{m},
\end{gathered}
$$

where $k=\omega / c$. The components of the electric field are 


$$
\begin{aligned}
E_{m}^{r,>}(r)= & \frac{i}{2 k^{2} r r_{0}}\left\{-c_{m}^{+}\left(2 m+2 m^{2}+k^{2} r^{2}\right)\left(\frac{r}{r_{0}}\right)^{m}\right. \\
& \left.+k^{2} r_{0}^{2}\left(c_{m}^{-}\left(\frac{r}{r_{0}}\right)^{m}-\frac{i e Z_{0}}{2 \pi r_{0}}\left(\frac{r_{0}}{r}\right)^{m}\right)\right\}, \\
E_{m}^{\phi,>}(r)= & \frac{1}{2 k^{2} r r_{0}}\left\{c_{m}^{+}\left(2 m+2 m^{2}-k^{2} r^{2}\right)\left(\frac{r}{r_{0}}\right)^{m}\right. \\
& \left.-k^{2} r_{0}^{2}\left(c_{m}^{-}\left(\frac{r}{r_{0}}\right)^{m}+\frac{i e Z_{0}}{2 \pi r_{0}}\left(\frac{r_{0}}{r}\right)^{m}\right)\right\}, \\
E_{m}^{z,>}(r)= & \frac{m+1}{k r_{0}}\left(\frac{r}{r_{0}}\right)^{m} c_{m}^{+} .
\end{aligned}
$$

For $r>a$, the equations in (8) can be rewritten adding to both sides of equations a term $(\omega / c)^{2}(1-\epsilon) B_{m}^{ \pm}$. In the ultrarelativistic case, the first equation in (8) for the case of $r>a$ takes the form

$$
\left[\frac{1}{r} \frac{\partial}{\partial r} r\left(\frac{\partial}{\partial r}\right)-\left(\frac{m \pm 1}{r}\right)^{2}+k_{w}^{2}\right] B_{m}^{ \pm}(r)=R_{m}^{ \pm}(r) .
$$

Here $k_{w}^{2}=\left(\frac{\omega}{c}\right)^{2}(\epsilon-1)$ is a constant given by the $\epsilon$ of the metal,

$$
k_{w}^{2}=i \frac{4 \pi \sigma}{\omega}\left(\frac{\omega}{c}\right)^{2}, \quad k_{w}=\frac{1+i}{\delta_{\omega}},
$$

and $\delta_{\omega}=c / \sqrt{2 \pi \sigma \omega}$ is the skin depth.

The RHS $R_{m}^{ \pm}(r)$ is equal to zero in the metal. In the slots, $r>a+\Delta,|\phi|<\alpha / 2$, the RHS is then

$$
\begin{aligned}
R_{m}^{ \pm, z}(r) & =k_{w}^{2} \sum_{n} \int_{-\alpha / 2}^{\alpha / 2} \frac{d \phi}{2 \pi} B_{n}^{ \pm, z}(r) e^{i(n-m) \phi} \\
& =\frac{\alpha k_{w}^{2}}{2 \pi} \sum_{n=-\infty}^{\infty} s(n-m) B_{n}^{ \pm, z}(r),
\end{aligned}
$$

where $\alpha$ is the angular slot width, and

$$
s(n-m)=\frac{\sin [(n-m) \alpha / 2]}{(n-m) \alpha / 2} .
$$

The solution of the homogeneous equation (16) at $r>a$ is given in terms of Bessel functions,

$$
\begin{aligned}
B_{m}^{0, \pm}(r)= & \alpha_{m}^{ \pm} H_{m \pm 1}^{(1)}\left(k_{w} r\right)+\beta_{m}^{ \pm} H_{m \pm 1}^{(2)}\left(k_{w} r\right) \\
B_{m}^{0, z}(r)= & -\frac{i k_{w}}{2 k}\left(\alpha_{m}^{+}-\alpha_{m}^{-}\right) H_{m}^{(1)}\left(k_{w} r\right) \\
& -\frac{i k_{w}}{2 k}\left(\beta_{m}^{+}-\beta_{m}^{-}\right) H_{m}^{(2)}\left(k_{w} r\right) .
\end{aligned}
$$

This solution is valid in the metal for $a<r<a+\Delta$, and the tangential components of the fields $B$ and $E=$ $(i / k \epsilon) \nabla \times B$ have to be matched with the solution inside of the beam pipe at the beam pipe radius $r=a$. For frequencies for which the skin depth $\delta_{\omega} \ll a$, we can use the asymptotic expression of the Bessel functions,

$$
\begin{aligned}
B_{m}^{0, \pm}(r)= & \sqrt{\frac{2}{\pi k_{w} r}}\left\{\alpha_{m}^{ \pm} e^{i\left[k_{w} r-(\pi / 2)(m \pm 1)-(\pi / 4)\right]}\right. \\
& \left.+\beta_{m}^{ \pm} e^{-i\left[k_{w} r-(\pi / 2)(m \pm 1)-(\pi / 4)\right]}\right\} \\
B_{m}^{0, z}(r)= & \frac{i k_{w}}{2 k} \sqrt{\frac{2}{\pi k_{w} r}}\left\{\left(\alpha_{m}^{+}-\alpha_{m}^{-}\right) e^{i\left[k_{w} r-(\pi / 2) m-(\pi / 4)\right]}\right. \\
& \left.+\left(\beta_{m}^{+}-\beta_{m}^{-}\right) e^{-i\left[k_{w} r-(\pi / 2) m-(\pi / 4)\right]}\right\}
\end{aligned}
$$

The solution of the inhomogeneous equation (16) for $r>a+\Delta$ can be obtained using Green's function $G_{m}^{ \pm}\left(r, r^{\prime}\right)$,

$$
\begin{aligned}
& B_{m}^{ \pm}(r)=B_{m}^{0, \pm}(r)+\int_{a+\Delta}^{\infty} r^{\prime} d r^{\prime} G_{m}^{ \pm}\left(r, r^{\prime}\right) R_{m}^{ \pm}\left(r^{\prime}\right) \\
& {\left[\frac{1}{r} \frac{\partial}{\partial r} r\left(\frac{\partial}{\partial r}\right)-\left(\frac{m \pm 1}{r}\right)^{2}+k_{w}^{2}\right] G_{m}^{ \pm}\left(r, r^{\prime}\right)=\frac{\delta\left(r-r^{\prime}\right)}{r}}
\end{aligned}
$$

Explicitly,

$$
\begin{aligned}
G_{m}^{ \pm}\left(r, r^{\prime}\right)= & -i \frac{\pi}{4} \theta\left(r-r^{\prime}\right)\left[H_{m \pm 1}^{(1)}\left(k_{w} r\right) H_{m \pm 1}^{(2)}\left(k_{w} r^{\prime}\right)\right. \\
& \left.-H_{m \pm 1}^{(1)}\left(k_{w} r^{\prime}\right) H_{m \pm 1}^{(2)}\left(k_{w} r\right)\right] .
\end{aligned}
$$

Here $\theta\left(r-r^{\prime}\right)$ is the step function, $\theta\left(r-r^{\prime}\right)=1$ for $r>$ $r^{\prime}$ and zero otherwise. Equation (22) then takes the form of the integral equation,

$$
\begin{aligned}
B_{m}^{ \pm}(r)= & B_{m}^{0, \pm}(r)-i \frac{\alpha k_{w}^{2}}{8} \sum_{n=-\infty}^{\infty} s(n-m) \\
& \times \int_{a+\Delta}^{r} r^{\prime} d r^{\prime}\left[H_{m \pm 1}^{(1)}\left(k_{w} r\right) H_{m \pm 1}^{(2)}\left(k_{w} r^{\prime}\right)\right. \\
& \left.-H_{m \pm 1}^{(1)}\left(k_{w} r^{\prime}\right) H_{m \pm 1}^{(2)}\left(k_{w} r\right)\right] B_{n}^{ \pm}\left(r^{\prime}\right) .
\end{aligned}
$$

Using asymptotic values for the Bessel functions and defining $b^{ \pm}(r)=\sqrt{r} B_{m}^{ \pm}(r)$, Eq. (24) for $r>a+\Delta$ takes the form

$$
\begin{aligned}
b_{m}^{ \pm}(r)= & \sqrt{r} B_{m}^{0, \pm}(r)+\frac{\alpha k_{w}}{2 \pi} \sum_{n=-\infty}^{\infty} s(n-m) \\
& \times \int_{a+\Delta}^{r} d r^{\prime} \sin \left[k_{w}\left(r-r^{\prime}\right)\right] b_{n}^{ \pm}\left(r^{\prime}\right) .
\end{aligned}
$$

For $r<a+\Delta, b_{m}^{ \pm}(r)=\sqrt{r} B_{m}^{0, \pm}(r)$.

In the case of a thick wall, $b_{m}^{ \pm}(r)$ has to decay at large $r$. If there are no slots, then $\beta_{m}^{ \pm}=0, B_{m}^{ \pm}(r)=B_{m}^{0, \pm}(r)$,

$$
\begin{aligned}
& B_{m}^{ \pm}(r)=\alpha_{m}^{ \pm} H_{m}^{(1)}\left(k_{w} r\right), \\
& B_{m}^{z}(r)=\frac{i k_{w}}{2 k}\left(\alpha_{m}^{+}-\alpha_{m}^{-}\right) H_{m}^{(1)}\left(k_{w} r\right) .
\end{aligned}
$$

For a beam pipe wall with a slot, the condition $\beta_{m}^{ \pm}=0$ is not valid because the integral term in Eq. (25) gives an exponentially growing contribution. Therefore, $\beta_{m}^{ \pm}$can be defined only after Eq. (25) is solved. 
To proceed further, we notice that, for a beam pipe with a slot, there are azimuthal harmonics with $m>0$ even for a beam with a zero offset. Such harmonics have the same magnitude at all symmetrically placed slots and we are not interested in such harmonics if the goal is to build a beam position monitor detecting the difference of the signals on the opposite wires. The signal in this case is given by the harmonics due to the nonzero beam offset. We can expect that such azimuthal harmonics $b_{n}^{ \pm}$get smaller for larger $n$. This is certainly the case when there are no slots. In this case, if the beam has a zero offset $r_{0}=0$ there is only the $n=0$ harmonics and with a small $r_{0}$ the harmonics $b_{n}^{ \pm} \propto$ $\left(r_{0} / a\right)^{n}$. For narrow slots such a hierarchy still exists although nonzero harmonics may be present even for the zero offset case. This allows us to use a perturbation technique taking into account only the lowest azimuthal harmonics and neglecting the coupling between higher order azimuthal harmonics. However, if the goal is to build a pickup, the following results can be used as an estimate to obtain the order of the signal.

Let us consider first the mode $m=0$ neglecting coupling to the nonzero modes. Equation (25) takes the form of the Volterra integral equation of the second kind:

$$
\begin{aligned}
b_{0}^{ \pm}(r)= & f_{0}^{ \pm}(r)+\frac{\alpha k_{w}}{2 \pi} \theta(r-a-\Delta) \\
& \times \int_{a+\Delta}^{r} d r^{\prime} \sin \left[k_{w}\left(r-r^{\prime}\right)\right] b_{0}^{ \pm}\left(r^{\prime}\right),
\end{aligned}
$$

where $f_{0}^{ \pm}(r)$ is the field in the beam pipe wall with no slots

$$
\begin{aligned}
f_{0}^{ \pm}(r)= & \sqrt{\frac{2}{\pi k_{w}}}\left\{\alpha_{0}^{ \pm} e^{i\left[k_{w} r \mp(\pi / 2)-(\pi / 4)\right]}\right. \\
& \left.+\beta_{0}^{ \pm} e^{-i\left[k_{w} r \mp(\pi / 2)-(\pi / 4)\right]}\right\} .
\end{aligned}
$$

Solution of Eq. (27) at $r>a+\Delta$ can be obtained using a Laplace transform, defining

$$
\begin{aligned}
\tilde{b}(p) & =\int_{a+\Delta}^{\infty} d r e^{-p r} b_{0}^{ \pm}(r), \\
\tilde{f}_{0}(p) & =\int_{a+\Delta}^{\infty} d r e^{-p r} f_{0}^{ \pm}(r) .
\end{aligned}
$$

Integrating by parts, we get

$$
\tilde{b}(p)=\frac{\tilde{f}_{0}(p)}{1-K(p)},
$$

where $K(p)$ is the Laplace transform of the kernel in Eq. (27),

$$
K(p)=\frac{\alpha k_{w}}{2 \pi} \int_{0}^{\infty} d r e^{-p r} \sin \left(k_{w} r\right)=\frac{\alpha}{2 \pi} \frac{k_{w}^{2}}{p^{2}+k_{w}^{2}} .
$$

The inverse Laplace transform gives at $r>a+\Delta$ :

$$
b_{0}^{ \pm}(r)=f_{0}^{ \pm}(r)+\int_{a+\Delta}^{r} d r^{\prime} R\left(r-r^{\prime}\right) f_{0}^{ \pm}\left(r^{\prime}\right),
$$

where

$$
R(r)=\int_{-i \infty+\varepsilon}^{i \infty+\varepsilon} \frac{d p}{2 \pi i} e^{p r} \frac{K(p)}{1-K(p)} .
$$

Here $\varepsilon>0$ and the contour is to the right of the integrand singularities.

Simple calculations give

$$
R(r)=\frac{\alpha k_{w}}{2 \pi \kappa} \sin \left[\kappa k_{w} r\right],
$$

where $\kappa=\sqrt{1-\alpha / 2 \pi}$.

Equation (32) then gives

$$
\begin{aligned}
b_{0}^{ \pm}(r)= & b_{g}(r)+b_{d}(r), \\
b_{g}(r)= & \frac{1}{\kappa \sqrt{2 \pi k_{w}}} e^{-i k_{w}(r-a-\Delta) \kappa-i k_{w}(a+\Delta) \mp i(\pi / 2)-i(\pi / 4)} \\
& *\left[\alpha_{0}^{ \pm}(-1+\kappa) e^{2 i k_{w}(a+\Delta)}\right. \\
& \left.+\beta_{0}^{ \pm}(1+\kappa) e^{2 i[ \pm(\pi / 2)+(\pi / 4)]}\right], \\
b_{d}(r)= & \frac{1}{\kappa \sqrt{2 \pi k_{w}}} e^{i k_{w}(r-a-\Delta) \kappa-i k_{w}(a+\Delta) \mp i(\pi / 2)-i(\pi / 4)} \\
& \times\left[\alpha_{0}^{ \pm}(1+\kappa) e^{2 i k_{w}(a+\Delta)}\right. \\
& +\beta_{0}^{ \pm}(-1+\kappa) e^{2 i[ \pm(\pi / 2)+(\pi / 4)]} .
\end{aligned}
$$

The term $b_{g}(r)$ grows exponentially with $r$ and has to be canceled out. That defines

$$
\beta_{0}^{ \pm}=i \alpha_{0}^{ \pm} \kappa_{0} e^{2 i k_{w}(a+\Delta)},
$$

where

$$
\kappa_{0}=\frac{1-\kappa}{1+\kappa}, \quad \kappa=\sqrt{1-\frac{\alpha}{2 \pi}} .
$$

Hence,

$$
\begin{aligned}
B_{0}^{ \pm}(r)= & \mp \alpha_{0}^{ \pm}(1+i) \sqrt{\frac{1}{\pi k_{w} r}}\left\{e^{i k_{w} r}+\frac{1-\kappa}{1+\kappa} e^{-i k_{w} r+2 i k_{w}(a+\Delta)}\right\}, \\
& (a<r<a+\Delta) \\
B_{0}^{ \pm}(r)= & \mp \alpha_{0}^{ \pm} \frac{(1+i)}{\sqrt{\pi k_{w} r}} \frac{2}{1+\kappa} e^{i k_{w}(r-a-\Delta) \kappa+i k_{w}(a+\Delta)}, \\
& (r>a+\Delta) .
\end{aligned}
$$

Note hat $B_{0}^{ \pm}(r)$ and its derivative are continuous at $r=$ $a+\Delta$.

Calculations of $B_{0}^{z}(r)$ give for $r<a+\Delta$

$B_{0}^{z}(r)=\frac{(1+i)}{2 k} \sqrt{\frac{k_{w}}{\pi r}}\left(\alpha_{0}^{+}-\alpha_{0}^{-}\right)\left(e^{i k_{w} r}-\kappa_{0} e^{-i k_{w} r+2 i k_{w}(a+\Delta)}\right)$,

For the harmonics $m>0$, calculations can be carried out in the similar way. For $m>0$ and $a<r<a+\Delta$, the solution is $b_{m}^{ \pm}(r)=f_{m}^{ \pm}(r)$, 


$$
\begin{aligned}
f_{m}^{ \pm}(r)= & \sqrt{\frac{2}{\pi k_{w}}}\left\{\alpha_{m}^{ \pm} e^{i\left[k_{w} r-(3 \pi / 4)-(\pi m / 2)\right]}\right. \\
& \left.+\beta_{m}^{ \pm} e^{-i\left[k_{w} r-(3 \pi / 4)-(\pi m / 2)\right]}\right\}
\end{aligned}
$$

with constants $\alpha_{m}^{ \pm}$and $\beta_{m}^{ \pm}$.

For $m>0$ and $r>a+\Delta$ and taking into account coupling to the $m=0$ mode, Eq. (25) gives

$$
\begin{aligned}
b_{m}^{ \pm}(r)= & h_{m}^{ \pm}(r)+\frac{\alpha k_{w}}{2 \pi} \theta(r-a-\Delta) \int_{a+\Delta}^{r} d r^{\prime} \\
& \times \sin \left[k_{w}\left(r-r^{\prime}\right)\right] b_{m}^{ \pm}\left(r^{\prime}\right),
\end{aligned}
$$

where

$$
\begin{aligned}
h_{m}^{ \pm}(r)= & f_{m}^{ \pm}(r)+\frac{\alpha k_{w}}{2 \pi} s(m) \theta(r-a-\Delta) \\
& \times \int_{a+\Delta}^{r} d r^{\prime} \sin \left[k_{w}\left(r-r^{\prime}\right)\right] b_{0}^{ \pm}\left(r^{\prime}\right) .
\end{aligned}
$$

Note that the solution of Eq. (40) is automatically matched with Eq. (39) at $r=a+\Delta$ with its derivative. The explicit form of the solution can be obtained with a Laplace transform similar to the method used in the $m=0$ case,

$$
\begin{aligned}
b_{m}^{ \pm}(r)= & h_{m}^{ \pm}(r)+\frac{\alpha k_{w}}{2 \pi \kappa} \theta(r-a-\Delta) \\
& \times \int_{a+\Delta}^{r} d r^{\prime} \sin \left[\kappa k_{w}\left(r-r^{\prime}\right)\right] h_{m}^{ \pm}\left(r^{\prime}\right) .
\end{aligned}
$$

Equation (32) can be obtained from here replacing $h_{m}^{ \pm}(r)$ with $f_{0}^{ \pm}(r)$. Equation (42) can be simplified using Eq. (32),

$$
\begin{aligned}
b_{m}^{ \pm}(r)= & f_{m}^{ \pm}(r)+\frac{\alpha k_{w}}{2 \pi \kappa} \theta(r-a-\Delta) \\
& \times \int_{a+\Delta}^{r} d r^{\prime} \sin \left[\kappa k_{w}\left(r-r^{\prime}\right)\right] f_{m}^{ \pm}\left(r^{\prime}\right) \\
& +\left(1-\delta_{m, 0}\right) s(m) \theta(r-a-\Delta) \frac{\alpha k_{w}}{2 \pi \kappa} \\
& \times \int_{a+\Delta}^{r} d r^{\prime} \sin \left[\kappa k_{w}\left(r-r^{\prime}\right)\right] b_{0}^{ \pm}\left(r^{\prime}\right) .
\end{aligned}
$$

To cancel the exponentially growing terms at $r \rightarrow \infty$, we put

$$
\beta_{m}^{ \pm}=i \kappa_{0}\left\{\alpha_{m}^{ \pm}+\frac{s(m)}{\kappa} \alpha_{0}^{ \pm} e^{i m \pi / 2}\right\} e^{2 i k_{w}(a+\Delta)-i \pi m} .
$$

That defines the fields in the wall in terms of the coefficients $\alpha_{m}^{ \pm}$and $c_{m}^{ \pm},>, m=0,1, \ldots$ These coefficients are determined by matching the tangential components of the $E$ and $B$ fields at $r=a$ for each $m$. Calculations are straightforward but cumbersome. Because the full expression is too long to produce here, we give the explicit result in the limit $k_{w} \gg k, k^{2} a / k_{w} \ll 1$ neglecting terms with additional factors $k / k_{w}$ or $1 /\left(k_{w} a\right)$. In this case,

$$
\begin{aligned}
c_{m}^{+}= & -\frac{e Z_{0}}{2 \pi a}\left(\frac{r_{0}}{a}\right)^{m+1}\left(\frac{k}{k_{w}}\right)^{2}\left(\frac{k_{w} a}{(1+m)\left(1+\kappa_{0} e^{2 i k_{w} \Delta}\right)}\right) \\
& \times\left\{\left(\frac{r_{0}}{a}\right)^{m}\left(1-\kappa_{0} e^{2 i k_{w} \Delta}\right)-e^{2 i k_{w} \Delta} \frac{s(m) \kappa_{0}}{(m+1) \kappa g_{0}}\right. \\
& \left.\times\left[2 i(m+1)-\frac{k^{2} a}{k_{w}}\left(1-\kappa_{0} e^{2 i k_{w} \Delta}\right)\right]\right\},
\end{aligned}
$$

$$
\begin{aligned}
c_{m}^{-}= & \frac{e Z_{0}}{2 \pi a}\left(\frac{r_{0}}{a}\right)^{m-1}\left\{-i\left(\frac{r_{0}}{a}\right)^{m}-\frac{s(m)}{k_{w} a g_{0}} \frac{\kappa_{0}}{\kappa}\right. \\
& \times\left(\frac{e^{2 i k_{w} \Delta}}{(1+m)\left(1-\kappa_{0} e^{2 i k_{w} \Delta}\right)}\right) \\
& \left.\times\left[\frac{k^{2} a}{k_{w}}+i\left[k^{2} a^{2}-2 m(m+1)\right]\left(1-\kappa_{0} e^{2 i k_{w} \Delta}\right)\right]\right\},
\end{aligned}
$$

$$
\begin{aligned}
\alpha_{m}^{+}= & (1+i) \frac{e Z_{0}}{4} \sqrt{\frac{k_{w}}{\pi a}} \frac{e^{-i k_{w} a+i m \pi / 2}}{1+\kappa_{0} e^{2 i k_{w} \Delta}}\left\{-\left(\frac{r_{0}}{a}\right)^{m}\right. \\
& \left.+e^{2 i k_{w} \Delta} \frac{\kappa_{0} s(m)}{\kappa g_{0}}\left[i+\frac{m}{k_{w} a}-\frac{k^{2} a}{(1+m) k_{w}}\right]\right\}, \\
\alpha_{m}^{-}= & \alpha_{m}^{+}\left(1-\frac{2 k^{2}}{k_{w}^{2}}\right)+\frac{(1+i) e Z_{0} k^{2} s(m)}{2 g_{0} k_{w}^{3} a^{2}\left(1-\kappa_{0} e^{2 i k_{w} \Delta}\right)^{2}}\left(\frac{\kappa_{0}}{\kappa}\right) \\
& \times \sqrt{\frac{k_{w} a}{\pi}} e^{-i k_{w} a+2 i k_{w} \Delta+i m \pi / 2} .
\end{aligned}
$$

where

$$
g_{0}=i\left(1+\kappa_{0} e^{2 i k_{w} \Delta}\right)+\frac{k^{2} a}{k_{w}}\left(1-\kappa_{0} e^{2 i k_{w} \Delta}\right) .
$$

The coefficients $\alpha_{0}^{ \pm}$for the harmonics $m=0$ can be obtained from here by putting $s(m) \rightarrow 0$ and then $m \rightarrow 0$.

\section{RESULTS}

Equations (39)-(46) define the fields in the wall and the beam pipe. For example, the azimuthal component of $B_{m}^{\phi}(r)$ in the range $a<r<a+\Delta$ is

$$
\begin{aligned}
B_{m}^{\phi}(r)= & \frac{e^{3 i \pi / 4+i k_{w} r}}{\kappa(1+\kappa)} \sqrt{\frac{1}{2 \pi k_{w} r}}\left\{-e^{-2 i k_{w}(r-a-\Delta)}(-1+\kappa)\right. \\
& \times s(m)\left(\alpha_{0}^{+}+\alpha_{0}^{-}\right) \\
& +\kappa e^{-i m \pi / 2}\left[-e^{-2 i k_{w}(r-a-\Delta)}(-1+\kappa)\right. \\
& \left.+(1+\kappa)]\left(\alpha_{m}^{+}+\alpha_{m}^{-}\right)\right\} .
\end{aligned}
$$

The field $B_{m}^{\phi}(r)=(-i / 2)\left(B_{m}^{+}-B_{m}^{-}\right)$in the range $r>$ $a+\Delta$ is defined by 


$$
\begin{aligned}
B_{m}^{ \pm}(r)= & \frac{(1+i)}{\kappa(1+\kappa)} \frac{e^{i k_{w}[a+\Delta+\kappa(r-a-\Delta)]}}{\sqrt{\pi k_{w} r}} \\
& \times\left\{ \pm i s(m)(\kappa-1)\left[-i-k_{w}(r-a-\Delta)(1+\kappa)\right]\right. \\
& \left.\times \alpha_{0}^{ \pm} e^{-i m \pi / 2} \mp 2 \kappa \alpha_{m}^{ \pm}\right\} .
\end{aligned}
$$

The radial dependence of the $m=1$ harmonics of $B^{\phi}(r)$ within the wall at a frequency of $1 \mathrm{MHz}$ is illustrated in
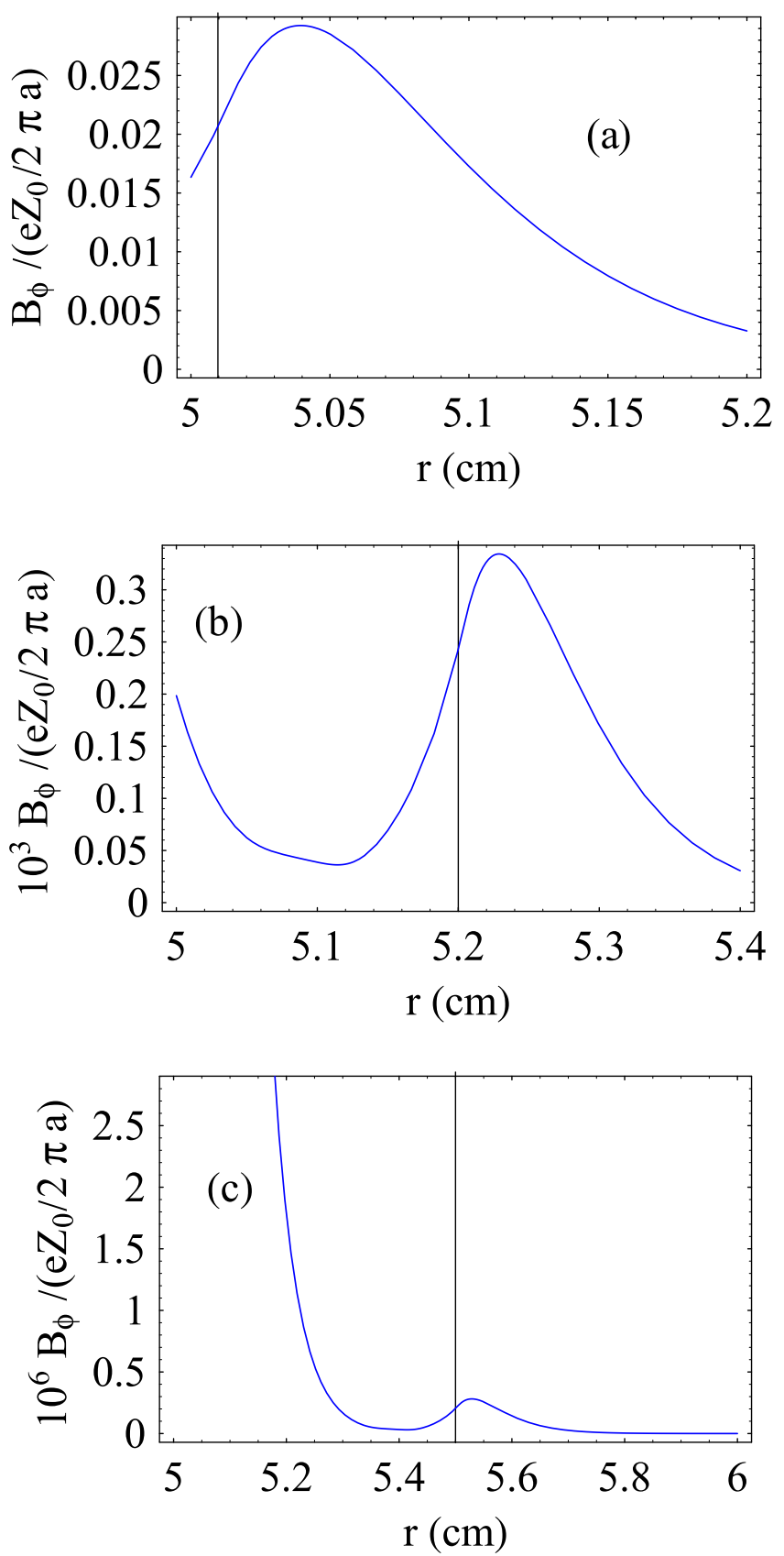

FIG. 5. (Color) Radial dependence of the $m=1$ harmonics of $B^{\phi}(r)$ within the wall for (a) $\Delta=0.01 \mathrm{~cm}$, (b) $\Delta=0.2 \mathrm{~cm}$, and (c) $\Delta=0.5 \mathrm{~cm}$. The vertical lines correspond to the radius $a+$ $\Delta$. Note the difference in scale. Other parameters are given in the text.
Fig. 5 for three values of the screen thickness (a) $\Delta=0.01 \mathrm{~cm}$, (b) $\Delta=0.2 \mathrm{~cm}$, and (c) $\Delta=0.5 \mathrm{~cm}$. The other parameters were: $a=5 \mathrm{~cm}$, stainless-steel wall conductivity $\sigma=1.4 \times 10^{4} \Omega^{-1} \mathrm{~cm}^{-1}, \alpha=0.04$, and the offset $r_{0}=0.001 \mathrm{~cm}$. At a frequency of $1 \mathrm{MHz} \delta_{\omega}=$ $0.042 \mathrm{~cm}$. The radial behavior shows the resonance character caused by reflection from the slot.

The magnetic flux $\Phi_{m}(\omega)$ due to the $m$ th harmonics through the wire contour with the length $L$ shown in Fig. 4 is obtained by integrating

$$
\begin{aligned}
\Phi_{m}(\omega)= & L \int_{a+\Delta}^{\infty} d r B_{m}^{\phi}(r) \\
= & \frac{L(1+i)}{2 k_{w} \kappa^{2}(1+\kappa)} \frac{e^{i k_{w}(a+\Delta)-i m \pi / 2}}{\sqrt{\pi k_{w}(a+\Delta)}} \\
& \times\left\{s(m) \frac{(\kappa-1)}{\kappa}\left(\alpha_{0}^{+}+\alpha_{0}^{-}\right) e^{i m \pi / 2}\right. \\
& \left.-2 \kappa\left(\alpha_{m}^{+}+\alpha_{m}^{-}\right)\right\} .
\end{aligned}
$$

$\Phi_{m}(\omega)$ defines the harmonics of the voltage $V_{m}(\omega)$ induced in the contour,

$$
V_{m}(\omega)=\oint E_{\omega} . d l=\left(\frac{i k}{2 \pi}\right) \Phi_{m}(\omega) .
$$

The spectral density is shown in Fig. 6.

Induced voltage $U_{m}(t)$ in the contour by a single beam particle lagging at $z_{i}$ from the bunch center is obtained by integrating over all frequencies

$$
U_{m}(t)=2 \operatorname{Re}\left[\int_{0}^{\infty} V_{m}(\omega) d \omega e^{-i \omega z_{i}-i \omega t} d \omega\right] .
$$

The signal from a bunch is obtained by summing up contributions of all particles. For a Gaussian bunch with an rms length $\sigma_{b}$ and population $N_{b}$, we replace the sum by a convolution of the spectrum density of the bunch,

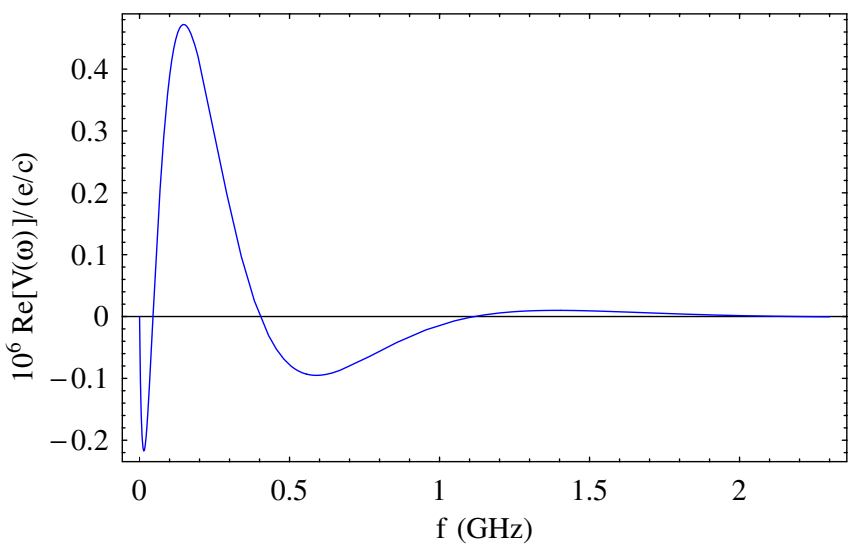

FIG. 6. (Color) Spectral density $V_{m}(\omega)$ of the dipole harmonics $m=1$ of the azimuthal $B^{\phi}$ field per single beam particle, $L=$ $10 \mathrm{~cm}$. Other parameters are the same as in Fig. 5. 


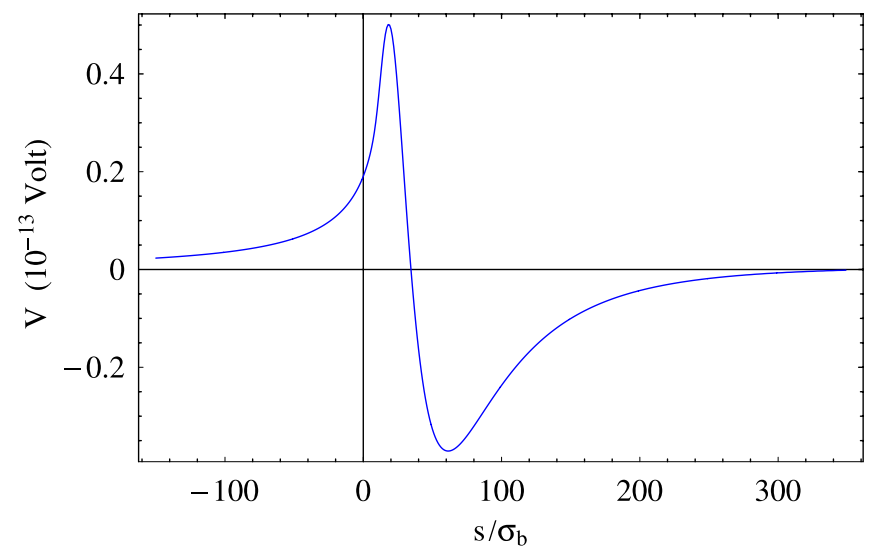

FIG. 7. (Color) Voltage induced in the wire loop by the dipole harmonics of the azimuthal $B^{\phi}$ field of a single beam particle, $\Delta=0.01 \mathrm{~cm}$. Other parameters are the same as in Fig. 5 .

$$
U_{m}^{\text {bunch }}(t)=2 N_{b} \operatorname{Re}\left[\int_{0}^{\infty} V_{m}(\omega) e^{-(1 / 2)\left(\omega \sigma_{b} / c\right)^{2}} e^{-i k s} d \omega\right]
$$

The result from integration of a single electron for $m=$ 1 mode is shown in Fig. 7 for $L=10 \mathrm{~cm}$ and $a=5 \mathrm{~cm}$.

The longitudinal beam impedance $Z_{m}^{l}(\omega)$ per unit length for a beam in the beam pipe is given by the coefficient $c_{m}^{+}$,

$$
\begin{aligned}
& Z_{m}^{l}(\omega)=-\frac{1}{e} \frac{(m+1) c_{m}^{+}}{k r_{0}}\left(\frac{r}{r_{0}}\right)^{m} \\
& Z_{m}^{l}(\omega)= \frac{Z_{0}}{2 \pi a}\left(\frac{r}{a}\right)^{m}\left(\frac{k}{k_{w}}\right)\left(\frac{1}{1+\kappa_{0} e^{2 i k_{w} \Delta}}\right) \\
& \times\left\{\left(\frac{r_{0}}{a}\right)^{m}\left(1-\kappa_{0} e^{2 i k_{w} \Delta}\right)-e^{2 i k_{w} \Delta} \frac{s(m) \kappa_{0}}{(m+1) \kappa g_{0}}\right. \\
&\left.\times\left[2 i(m+1)-\frac{k^{2} a}{k_{w}}\left(1-\kappa_{0} e^{2 i k_{w} \Delta}\right)\right]\right\}
\end{aligned}
$$

For $r_{0} \ll a$ the longitudinal impedance is dominated by the contribution of the $m=0$ mode. Neglecting terms $k^{2} a \delta(\omega) \ll 1$ and for $\Delta \gg \delta(\omega)$ we have

$$
Z^{l}(\omega)=\frac{Z_{0}}{2 \pi a}\left(\frac{k}{k_{w}}\right)\left(\frac{1-\kappa_{0} e^{2 i k_{w} \Delta}}{1+\kappa_{0} e^{2 i k_{w} \Delta}}\right) .
$$

In the limit that $\Delta \rightarrow \infty$ Eq. (58) gives the usual result for the longitudinal impedance per unit length

$$
Z^{l}(\omega)=(1-i) \frac{Z_{0}}{2 \pi a}\left(\frac{k \delta(\omega)}{2}\right)
$$

For an open slot $\Delta \rightarrow 0$ and small $\alpha \ll 1, k^{2} a \delta(\omega) \ll$ 1, Eq. (57) gives

$$
Z^{l}(\omega)=(1-i) \frac{Z_{0}}{2 \pi a}\left(\frac{k \delta(\omega)}{2}\right)\left(1-\frac{3 \alpha}{8 \pi}\right) .
$$

The dominant contribution to the transverse impedance per unit length is related to the longitudinal dipole impedance $m=1$ by the Panofsky-Wenzel theorem.

For small $\alpha \ll 1$,

$$
\begin{aligned}
Z^{\operatorname{tr}}(\omega)= & (1-i) \frac{Z_{0} \delta(\omega)}{4 \pi a^{3}}\left\{\frac{1-\kappa_{0} e^{2 i k_{w} \Delta}}{1+\kappa_{0} e^{2 i k_{w} \Delta}}-\frac{\kappa_{0}}{2 \kappa g_{0}}\left(\frac{a}{r_{0}}\right)\right. \\
& \left.\times \frac{e^{2 i k_{w} \Delta}}{1+\kappa_{0} e^{2 i k_{w} \Delta}}\left[4 i-\frac{k^{2} a}{k_{w}}\left(1-\kappa_{0} e^{2 i k_{w} \Delta}\right)\right]\right\} .
\end{aligned}
$$

For large $\Delta \gg \delta(\omega)$, the expression in the curly brackets is equal to one giving us the usual transverse resistive wall impedance per unit length. Equation (61) shows that, contrary to the usual resistive wall impedance, $Z^{\operatorname{tr}}(\omega)$ for a beam pipe with a slot depends on the offset $r_{0}$. This dependence is weak provided

$$
\frac{r_{0}}{a} \gg \frac{\alpha}{4 \pi} e^{-2 \Delta / \delta},
$$

but gives

$$
Z^{\operatorname{tr}}(\omega)=(1-i) \frac{Z_{0} \delta(\omega)}{4 \pi a^{3}}\left[1-\frac{\alpha}{4 \pi}\left(\frac{a}{r_{0}}\right)\right]
$$

for an open slot $\Delta \rightarrow 0$. The second term in the impedance equation (63) corresponds to a constant force acting on the beam due to a longitudinal slot in the beam pipe. Calculating the wakefield due to the last term in Eq. (63), we got

$$
\begin{aligned}
& W_{\mathrm{tr}}(z)=i \int \frac{d \omega}{2 \pi} Z^{\operatorname{tr}}(\omega) e^{-i \omega z / c} \\
& W_{\mathrm{tr}}(z)=-\frac{\alpha}{2 \pi a^{2} r_{0}} \frac{1}{\sqrt{\pi Z_{0} \sigma z}}
\end{aligned}
$$

and using the equation of motion for a particle located at a distance $z$ from the head of a bunch with a bunch population $N_{b}$

$$
\frac{d^{2} x}{d s^{2}}+g(s) x(s)=\frac{N_{b} r_{e}}{\gamma} W_{\mathrm{tr}}(z) r_{0},
$$

we get for the average perturbation of the closed orbit to be

$$
\langle x(s)\rangle=-\frac{N_{b} r_{e}}{\gamma}\left(\frac{\alpha}{2 \pi}\right)\left\langle\left(\frac{\beta_{x}}{a}\right)^{2}\right\rangle \frac{1}{\sqrt{\pi Z_{0} \sigma z}} .
$$

Here $r_{e}$ is the classical electron radius, and $\beta_{x}$ is the horizontal beta function. The effect is very small, $\langle x(s)\rangle \simeq$ $0.5 \mu \mathrm{m}$ for a particle at a distance of $z=1 \mathrm{~cm}$ for parameters $N_{b}=6.0 \times 10^{10}, \gamma=6.0 \times 10^{3}, \beta_{x}=15 \mathrm{~m}$, $a=5 \mathrm{~cm}$, and stainless-steel conductivity.

\section{ACKNOWLEDGMENTS}

The work of A.N. and S.H. was supported by Department of Energy Contract No. DE-AC0376SF00515. The work of A.A. was supported by 
Department of Energy Contract No. DE-AC0596OR22464.

[1] B. Zotter and S. Kheifets, Impedances and Wakes in High-Energy Particle Accelerators (World Scientific, Singapore, 1998).
[2] A. Novokhatski (to be published).

[3] SLAC Report No. SLAC-R-593, 2002.

[4] A. A. Mikhailichenko, Pribory i technika experimenta (Nauka, Moscow, 1984), Vol. N2, p. 149 (in Russian).

[5] A. V. Aleksandrov, Diploma thesis, Institute of Nuclear Physics, Novosibirsk, 1989. 